\title{
The Resilience of Microbial Community Involved in Coking Wastewater Treatment System
}

\author{
Shang Zhu ${ }^{1}$, Haizhen $\mathbf{W u}^{2}$, Lin Zhou ${ }^{1}$ and Chaohai Wei ${ }^{3^{*}}$
}

${ }^{1}$ Guangdong Province Key Laboratory for Biotechnology Drug Candidates, School of Biosciences and Biopharmaceutics, Guangdong Pharmaceutical University, Guangzhou 510006, PR China

${ }^{2}$ College of Biological Science and Engineering, South China University of Technology, Guangzhou 510006, PR China

${ }^{3}$ School of Environment and Energy, South China University of Technology, Guangzhou 510006, PR China

"Corresponding author: Chaohai Wei, School of Environment and Energy, South China University of Technology, Guangzhou 510006, PR China, Tel: +862039380588; Fax: +862039380588; E-mail: cechwei@scut.edu.cn

Rec date: Feb 28, 2017; Acc date: Mar 28, 2017; Pub date: Mar 30, 2017

Copyright: $\odot 2017$ Zhu S, et al. This is an open-access article distributed under the terms of the creative commons attribution license, which permits unrestricted use, distribution, and reproduction in any medium, provided the original author and source are credited.

\begin{abstract}
Biological treatment processes are the most important step for removing various nutrient matters due to their high removal efficiency and low operational cost. The activated sludge technology was used extensively in coking wastewater biological treatment processes where a complex prokaryotic microbial consortium played an important role in the processes of oxygen-depleting organics, toxics and nutrients removal and preventing discharge of pathogens. Thus, a thorough knowledge of the ecology of the coking wastewater activated sludge communities is required to improve system operation and stability. Therefore, there is necessary to reveal the resilience of microbial community involved in coking wastewater treatment system.
\end{abstract}

Keywords: Biological treatment processes; Nutrient matters; Wastewater; Pathogens; Microbial community

\section{Mini Review}

In this report, we investigated a full-scale coking wastewater treatment system with two paralleled units to see the resilience of microbial community. The full-scale CWW treatment system, an ongoing three-phase fluidized bed biological coking wastewater treatment system in Shaoguan, Guangdong Province of China, was run with south and north two paralleled units [1-4]. These two paralleled systems have been operating for more than years at daily capacity of approximately $1680 \mathrm{~m}^{3} \pm 80 \mathrm{~m}^{3}$. The total hydraulic retention time (HRT) was $46 \mathrm{~h}$. These two paralleled systems were able to maintain a stable process performance during operation and also the final effluent meet the national stringent effluent standards. The coking wastewater activated sludge of anaerobic process was collected from the corresponding bioreactor of two paralleled systems in March 2016. Illumina MiSeq high throughput sequencing technology was performed to reveal the relative abundance and diversity of microbiology in these two parallel systems. Since coking wastewater is usually regarded as an extreme environment with high toxicity and refractoriness, the microorganisms involved should be quite different from those in domestic wastewater [5]. But the microbial community which is responsible for the cooking wastewater treatment process is still significantly different?

The composition of the PCR products of V4 region of 16S rRNA genes was determined by MiSeq Sequencing. After MiSeq Sequencing. The raw data were processed and analyzed following the pipelines of Mothur and QIIME Raw data were denoised using the Mothur implementation of PyroNoise algorithm. Chimeric sequences were identified and removed using UCHIME with de novo method. Quality sequences were assigned to samples according to their unique 8-bp barcodes. Operational taxonomic units (OTUs, defined at the $97 \%$ sequence similarity level) were picked using average neighbor method after the Needleman alignment and a single-linkage precluster procedure. Taxonomy classification was identified using RDP Classifier at the $80 \%$ confidence threshold by default. Alpha- (number of OTUs, Chao 1, and Simpson and Shannon indices) diversity were statistically analyzed via rarefaction. Good's coverage estimators representing the subsample coverage were calculated using the Mothur software [6].

Functional stability of the two paralleled systems were defined and quantified by the CWW concentration of 12 items, which include COD, BOD, Phenols, Cyanides, Thiocyanate, ammonia, TN, TP, TSS, TOC, Sulfides, Oil. The COD removal during the operation period was approximately $92 \%$ with the average COD concentrations in the biological influent of less than $100 \mathrm{mg} / \mathrm{L}$. The phenol removal efficiency was approximately $99.3 \%$ in the final biological effluent. The ammonium removal reached nearly $91.2 \%$, indicating the good performance of ammonium-oxidizing bacteria (AOB) and nitriteoxidizing bacteria (NOB) in the two paralleled coking wastewater treatment systems. Almost all SCN-was completely removed in the aerobic bioreactor. The results showed that this industrial-scale two Paralleled coking wastewater treatment systems yielded stable contaminants removal efficiency with satisfactory performance. Temperatures for the two paralleled systems were relatively constant between $22^{\circ} \mathrm{C}$ to $27^{\circ} \mathrm{C}$ in the long-term performance. As reflected by the coking wastewater quality, the microbial community in different bioreactor kept adjusting to the wastewater characteristic and operation conditions.

There are some minor microbial community differences in the two parallel operation systems but will not affect their function, which is the resilience in the coking wastewater microbial community. We used MiSeq sequencing to analyze the microbial 16S rRNA genes across the parallel coking wastewater activated sludge samples. To understand the microbial community structure and function in the CWW biological treatment process, the classified OTUs were analyzed at phylum and 
Citation: Zhu S, Wu H, Zhou L, Wei C (2017) The Resilience of Microbial Community Involved in Coking Wastewater Treatment System. Next Generat Sequenc \& Applic 4: 142. doi:10.4172/2469-9853.1000142

Page 2 of 2

genus level. A total of 8 major (relative abundance $>1 \%$ within a sample) bacterial phyla (including four subphyla in the Proteobacteria) were identified. From the major phylum assignment result, it was found that the bacterial diversity in the two parallel systems was not significantly different from each other. The major phylum included $\gamma$ Proteobacteria, $\beta$-Proteobacteria, $\alpha$-Proteobacteria, $\zeta$-Proteobacteria, Acidobacteria, Actinobacteria, Bacteroidetes and Firmicutes. In order to further analyze the changes of the bacterial community involved in the biological treatment process of coking wastewater, besides the phylum, bacterial diversity and abundance were also analyzed more specifically at genus taxonomic units. A total of 10 major genera were captured the similarities and differences between the two parallel microbial communities at the genus level. The major genus included Pseudomonas, Comamonas, Alkaliphilus, Campylobacter, Pusillimonas, Petrimonas, Gp4, Corynebacterium, Ochrobactrum and Thiobacillus. In a word, no significant differences in the relative abundance of major genera among the two parallel systems were observed.

We pointed out the resilience of microbial communities in the parallel coking wastewater treatment systems at phylum and genus level. The diverse and distinct microbial community involved in biological processes of cooking wastewater indicating water characteristics and operational parameters determine the microbial community composition and function.

\section{Acknowledgement}

This works was supported by the National Natural Science Foundation of China (21377040 and 21207021) and the Program for
Science \& Technology of Guangdong Province, China (project No. 2015A020215008, 2016A020221037 and 2015B020235005).

\section{References}

1. Zhu XB, Tian JP, Liu C, Hen LJ (2013) Composition and dynamics of microbial community in a zeolite biofilter-membrane bioreactor treating coking wastewater. Appl Microbiol Biotechnol 97: 8767-8775.

2. Bai Y, Sun Q, Xing R, Wen D, Tang X (2011) Analysis of denitrifier community in a bioaugmented sequencing batch reactor for the treatment of coking wastewater containing pyridine and quinoline. Appl Microbiol Biotechnol 90: 1485-1492.

3. Zhang J, Wen DH, Zhao C, Tang XY (2014) Bioaugmentation accelerates the shift of bacterial community structure against shock load: A case study of coking wastewater treatment by zeolite-sequencing batch reactor. Appl Microbiol Biotechnol 98: 863-873.

4. Shade A, Peter H, Allison SD, Baho DL, Berga M, et al. (2012) Fundamentals of microbial community resistance and resilience. Front Microbiol 3: 417.

5. Bai Y, Sun Q, Sun R, Wen D, Tang X (2011) Bioaugmentation and adsorption treatment of coking wastewater containing pyridine and quinoline using zeolite-biological aerated filters. Environ Sci Technol 45: 1940-1948.

6. Schloss PD, Westcott SL, Ryabin T, Hall JR, Hartmann M, et al. (2009) Introducing mothur: Open-source, platform-independent, community supported software for describing and comparing microbial communities. Appl Environ Microbiol 75: 7537-7541. 